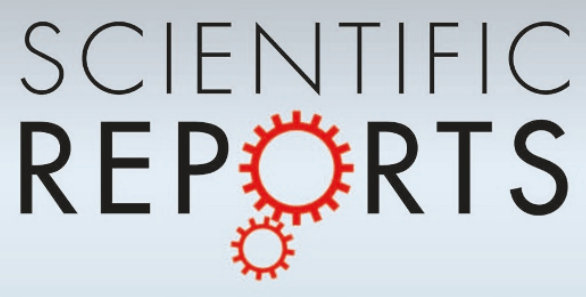

\section{OPEN}

SUBJECT AREAS:

IMMUNOPATHOGENESIS

BACTERIAL STRUCTURAL BIOLOGY

\section{Received \\ 23 October 2013 \\ Accepted \\ 2 January 2014 \\ Published \\ 22 January 2014}

Correspondence and requests for materials should be addressed to

A.M.C. (amc@ciad. $\mathrm{mx}$ )

\title{
Fecal microbiota imbalance in Mexican children with type 1 diabetes
}

\author{
María Esther Mejía-León ', Joseph F. Petrosino², Nadim Jose Ajami ${ }^{2}$, María Gloria Domínguez-Bello ${ }^{3,4}$ \\ \& Ana María Calderón de la Barca'
}

\begin{abstract}
'Department of Nutrition and Metabolism, Centro de Investigación en Alimentación y Desarrollo, A.C., Hermosillo, Sonora, México, ${ }^{2}$ Alkek Center for Metagenomics and Microbiome Research, Department of Molecular Virology and Microbiology, Baylor College of Medicine, Houston, Texas, USA, ${ }^{3}$ Laboratory of Microbial Ecology, Department of Biology, University of Puerto Rico, San Juan, Puerto Rico, ${ }^{4}$ Department of Medicine, New York University School of Medicine, New York, NY, USA.
\end{abstract}

Dysbiosis of the intestinal microbiota affecting the gut barrier could be triggering Type 1 Diabetes (T1D), the second most frequent autoimmune disease in childhood. This study compared the structure of the fecal microbiota in 29 mestizo children aged 7-18 years, including 8 T1D at onset, 13 T1D after 2 years treatment, and 8 healthy controls. Clinical information was collected, predisposing haplotypes were determined; the fecal DNA was extracted, the V4 region of the 16S rRNA gene amplified and 454-pyrosequenced. The newly diagnosed T1D cases had high levels of the genus Bacteroides $(\mathrm{p}<0.004)$, whereas the control group had a gut microbiota dominated by Prevotella. Children with T1D treated for $\geq 2$ years had levels of Bacteroides and Prevotella compared to those of the control group. The gut microbiota of newly diagnosed T1D cases is altered, but whether it is involved in disease causation or is a consequence of host selection remains unclear.

$\mathrm{T}$

ype 1 Diabetes (T1D) is a pro-inflammatory autoimmune disorder that results in $\beta$-cells destruction, leading to insulin deficiency ${ }^{1}$. T1D is one of the most frequent autoimmune disorders in childhood, with a worldwide prevalence of $1: 200$ to $1: 300$, which is higher than that reported a few decades ago ${ }^{2}$. The increasing incidence cannot be explained only by host genetic factors (HLA DR4-DQ8 and DR3-DQ2) ${ }^{3}$, and could instead reflect changes in early childhood exposure to risk factors ${ }^{4}$, such as lack of breastfeeding ${ }^{5}$ and viral infections ${ }^{6}$. Reported protection factors include microbial exposure $\mathrm{e}^{7,8}$ and selective antibiotic treatments in early life $\mathrm{e}^{7,9}$.

Associations between the intestinal microbiota alterations and B-cell autoimmunity have been described in HLA-DR/DQ genetically predisposed humans with positive GAD/IA2 autoantibodies ${ }^{10,11}$ or with T1D diagnosis $^{12}$. Autoimmunity can be triggered after the translocation of microbes through the intestinal epithelium as a result of increased permeability ${ }^{13}$. Interestingly, the pancreas and gut endoderm share a common embryonic origin, which may create an immune link between both organs ${ }^{14}$.

The aim of this study was to evaluate the structure of the gut microbiota in genetically predisposed Mexican children with T1D at onset, and after treatment for more than 2 years.

\section{Results}

Study population. All subjects were Sonoran by birth, with at least $90 \%$ of the parents and grandparents born in northwest Mexico. A total of 21 T1D cases and 8 healthy controls were included. Eight cases were newly diagnosed T1D cases (less than 2 months evolution) and 13 were controlled with insulin treatment (with more than 2 years evolution [T1D treated for $\geq 2$ years]) and HbA1C levels $<8 \%$. Four subjects in the control group were siblings of T1D cases. The subjects' mean age for the 3 groups was statistically similar (Table S1).

Clinical information. The number of antibiotic treatments per year, previous to diagnosis, was significantly higher $(p=0.043)$ in the T1D cases when compared with the antibiotic frequency in the same period in the controls (Table S1). The mean of antibiotic cycles per year in the newly diagnosed T1D cases was 4 cycles, while the T1D treated for more $>2$ year treatment group and controls had 6 and 3 cycles per year, respectively. At the time of the study, all subjects were on antibiotic-free scheme for at least 3 months prior to sampling. Other variables such as delivery mode, breastfeeding time, lactation regimes and infections per year, were not statistically different between controls and T1D cases $(p \geq 0.05)$. 
HLA types. All of the T1D cases (Table S1) had at least one HLA risk allele. The HLA DR3-DQ2 (DRB1*03/DQA1*0501/DQB1*0201) haplotype was in $19 \%$ of the cases, followed by $14.3 \%$ HLA DR4-DQ8 (DRB1*04/DQA1*0301/DQB1*0302/3) (Table S1). In addition, another $9.52 \%$ were heterozygous for the HLA DQ2DQ8 haplotype. Furthermore, 75\% of HLA DR3-DQ2 cases of T1D, were carriers of a DQ8 allele. Regarding DR alleles, $95 \%$ of the T1D children presented the DR4 variant (DRB1*04), while $66.6 \%$ were carriers of the DR3 allele (DRB1*03). In the control group, $12.5 \%$ were HLA DQ2. The rest of the control subjects were negative to DQ2 or DQ8; however, $37.5 \%$ presented isolated alleles associated with DQ8. All the control children were negatives for the DR alleles.

Gut microbiota. A total of 245,134 rRNA16S reads were obtained from the 29 samples, averaging 8,452 sequences per sample. The bacterial diversity was not significantly different between controls and the two T1D groups (Figures 1A, S1-2). However, beta diversity analysis using principal coordinates indicate a separate clustering of the fecal communities in subjects with T1D at onset compared to controls (Figure 1B). The group of T1D cases treated for 2 years did not show clustering and were spread along PC1 axis (Figures 1, 2, S3). Weighted UniFrac distances within groups were significantly smaller than between groups $(p=0.000001)$ thus indicating group clustering (Figure 2).

The major taxa explaining this clustering were not reflected at the phyla but at the genus level (Figure 3). Control children had a fecal community dominated by Prevotella, while children with T1D at onset had higher representation of Bacteroides $(p=0.0037)$ showing substantially reduced proportions of Prevotella $(p=0.0003)$,

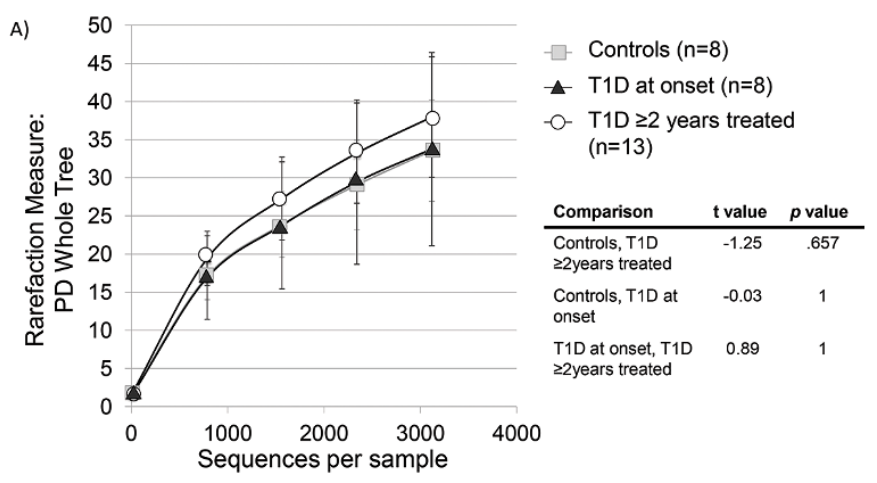

B)

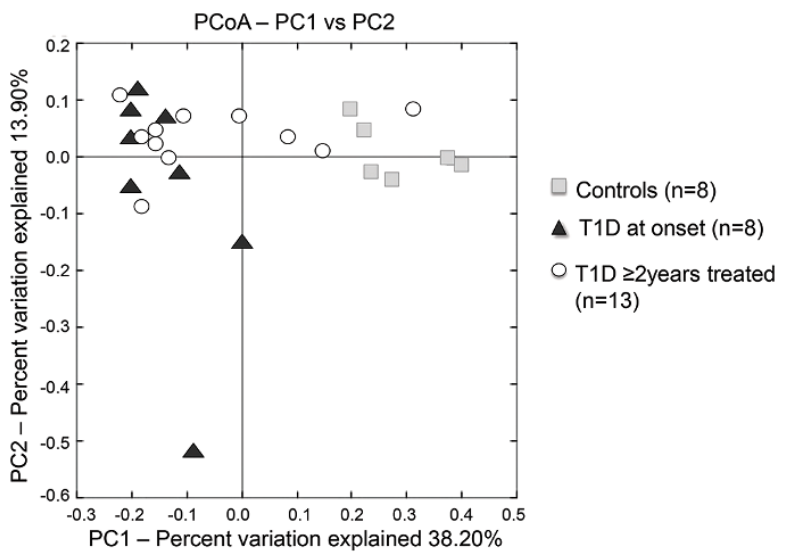

Figure $1 \mid$ Diversity of the microbiota in T1D and healthy children. (A) Rarefaction curves of phylogenetic diversity in fecal samples (Student's t test); (B) Principal Coordinate Analysis of fecal communities weighted Unifrac distances in T1D and healthy children.

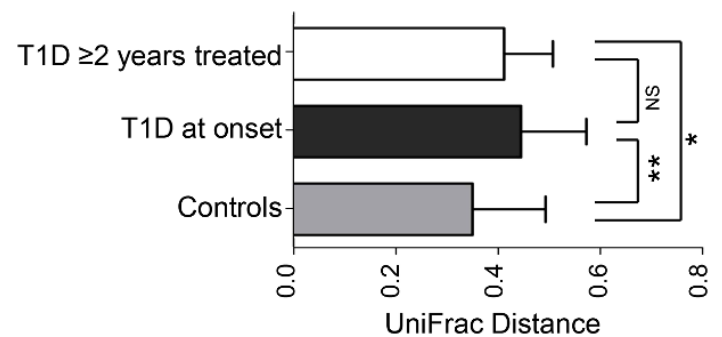

Figure $2 \mid$ Weighted UniFrac distances between groups. * $\mathrm{p}, 0.00005$, **p,0.000005, NS: non-significant (Student's t test).

Megamonas ( $p=0.0161)$ and Acidaminococcus $(p=0.0214)$, in comparison to controls. Children with T1D treated for 2 years showed an intermediate relative abundance of these genera between the controls and T1D new cases (Figure 3, S4, S5). Dominant phyla were Bacteroidetes and Firmicutes, followed by Proteobacteria and Tenericutes, with no differences between groups $(p>0.05)$, and thus the ratio Bacteroidetes/Firmicutes was not significantly different between our groups.

\section{Discussion}

T1D occurs typically in childhood or adolescence, and has been associated to host genetic factors with major histocompatibility complex (MHC) region harboring genes that contribute more than $50 \%$ of the risk, mediated by HLADR3-DQ2 or DR4-DQ8 ${ }^{15}$.

Our results show that these in T1D Mexican children had higher proportions of HLA DR4 (95\%) and DR3 (66.6\%) than MexicanAmerican T1D children ( $46 \%$ and $27 \%$ respectively) ${ }^{16}$. In addition to genetic factors, environmental factors have also been epidemiologically associated to T1D. These include early life factors such as Csection $^{17}$, lack of breastfeeding ${ }^{5}$ and early infections and antibiotic exposure ${ }^{7}$, all factors that are known to alter microbial communities ${ }^{18,19}$. The higher frequency of antibiotic treatments prior to diagnosis in T1D patients could influence the shift of the communities away from the controls; however, T1D at onset group had received fewer courses than the group of T1D treated for 2 years, and yet, had the most distinct community.

There was higher dispersion in the structure of the gut microbiota in T1D cases, than in controls (Figure 2). This finding is consistent with those found in previous studies related to autoimmunity and T1 $\mathrm{D}^{10,12}$, suggesting instability of the microbiome associated with this condition.

The fecal microbiota in Mexican healthy children of our study had a similar composition to that reported in people from developing countries with high levels of Prevotella, in contrast with the Bacteroides-dominated microbiota of US children and adults ${ }^{20,21}$. These differences are likely to be dietary, since Bacteroides has been associated with high protein and fat diets, while carbohydrate rich diets, increase Prevotella ${ }^{22}$. It is not clear if high dominance of Bacteroides in the US is associated with higher risk of TD1, which is indeed higher in US than in Mexican children (11 to 17 vs 1.5 per $100,000$, respectively $)^{23}$. The incidence of T1D in the Sonora region near the US-Mexican border- has doubled in the last 10 years $^{24}$, suggesting that the factors underlying risks are environmental, and could be related to diet or lifestyle changes.

Bacteroides-dominant gut communities were also observed in prediabetic Finish children who also showed decreased levels of Prevotella when compared to healthy controls $s^{25}$. Insulin treatment of T1D partially normalizes the microbiota profile towards Prevotella-dominant profile, indicating that physiological changes related to T1D are driving the gut microbiota structure in T1D. It has been suggested that Bacteroides activity in mucine-synthesis and degradation might be contributing to T1D development by thinning of the mucus layer, leading to increased gut permeability and inflammation ${ }^{26}$. Lactate 


\section{A)}

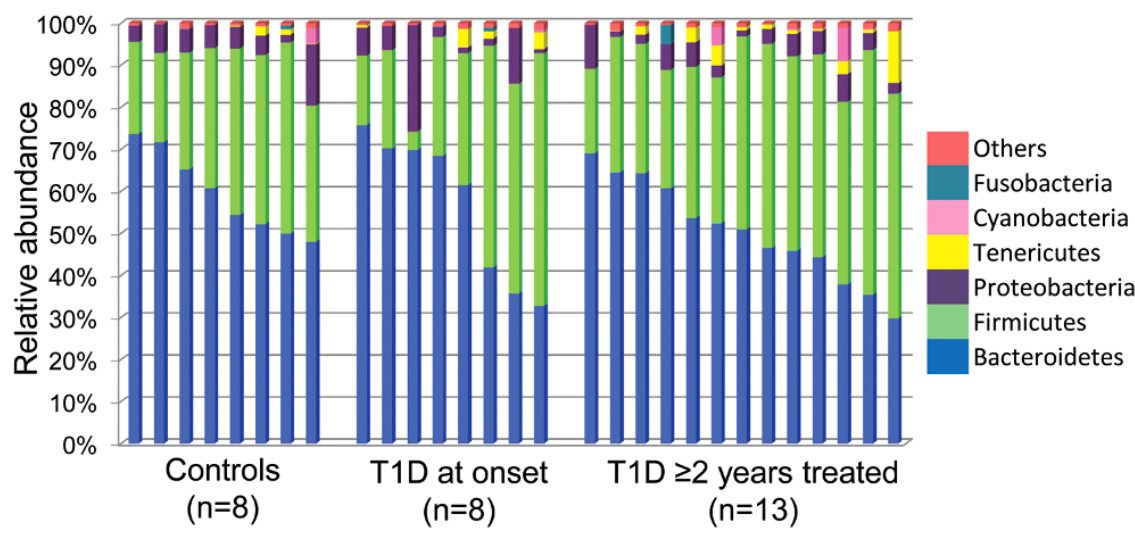

B)

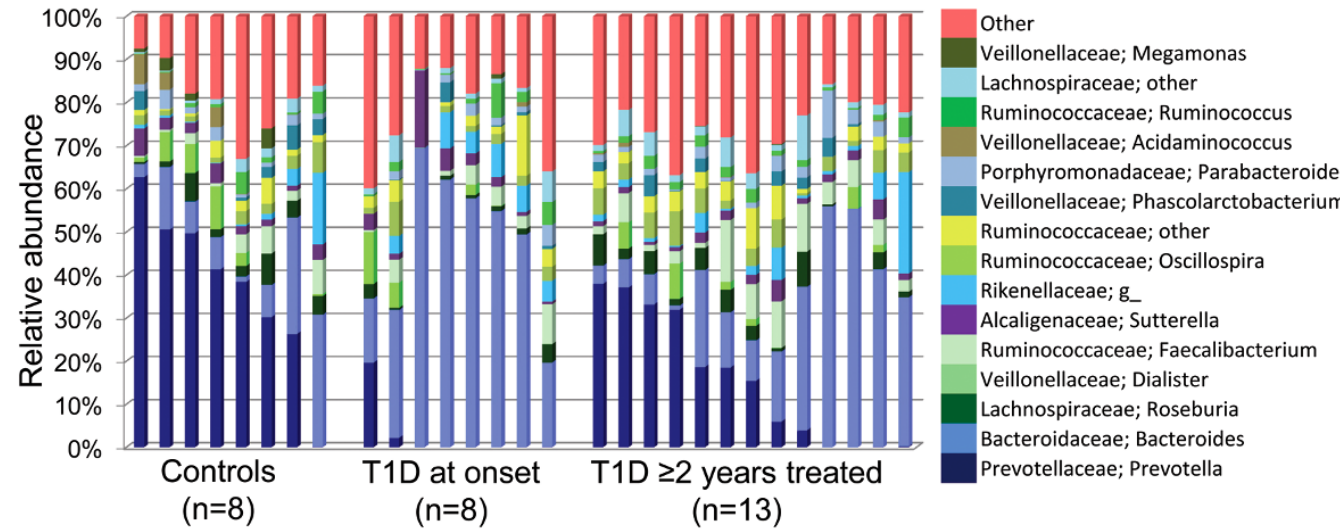

C)

Controls
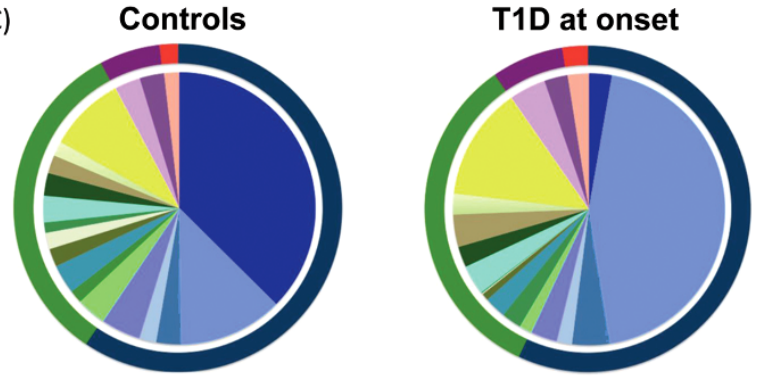

T1D $\geq 2$ years treated

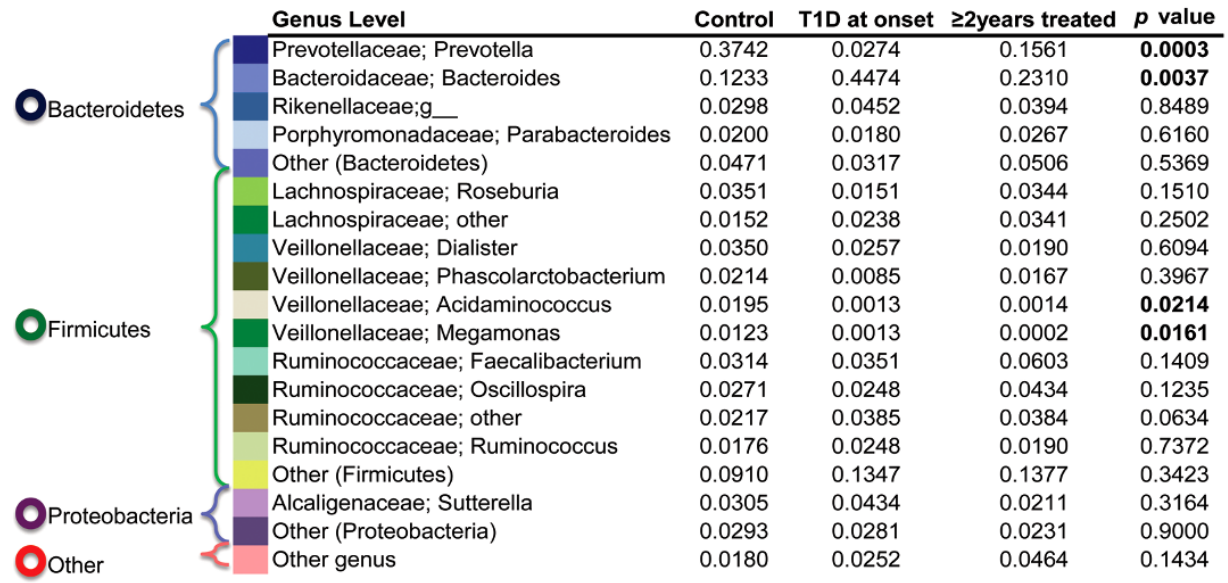

Figure 3 Average relative bacterial abundance of taxa in the fecal microbiota of healthy or T1D children. Relative abundance of fecal bacterial phyla (A) or genera (B) in each subject. Pie charts (C) depict phylum (outer ring) and genus (pie) level distribution. The proportions of the genus relative abundance are showed in the table and the $p$ values of ANOVA, Tukey-Kramer test refer to the comparison between the 3 groups. In this analysis only phyla and genera with relative abundance greater than $1 \%$ were included. All OTUs with lower abundance were grouped as "other". 
conversion to butyrate in the gut induces synthesis of mucins and consequently favors a healthy epithelium. Bacteroides would transform lactate into short chain fatty acids, such as propionate, acetate and succinate, which contribute to decrease mucin synthesis and therefore the tight junctions could be affected ${ }^{25}$. Thus, the lactate model seems to be critical to maintain intestinal health and explain the way to autoimmunity in T1D.

Bacterial antigens and microorganisms toxins can be sensed by molecules related to epithelial cells' tight junctions like zonulin, claudin and occludin, altering their activity and consequently increasing gut permeability and bacterial translocation ${ }^{27}$. In this context, some Bacteroides sp. with pathogen activity, such as Bacteroides fragilis, disrupts the tight junctions by proteolytic degradation due to metalloprotease enterotoxins (e.g. fragilysin) increasing paracellular permeability with local inflammation, cell damage and loss of microvilli $i^{28}$. This process could lead to loss of self-tolerance, causing aberrant immune responses, with an homeostatic imbalance of Tcells, gut inflammation and extra intestinal inflammatory infiltrate «insulitis» associated with $\mathrm{T}_{1} \mathrm{D}^{29}$.

The microbiota profile associated to T1D supports the idea of the existence of an "autoimmune" microbiome associated with diabetes ${ }^{10}$. However, this association does not necessarily imply causality. Intervention studies where the gut microbiota structure could be normalized in children at high risk for T1D via fecal transplant, have not yet been performed, but it could demonstrate an etiologic role of dysbiosis in T1D.

\section{Methods}

Study population. A cross-sectional case-control study was conducted in the Mexican Sonora state, between 2010 and 2012, recruiting children T1D patients at the Children's Hospital of the State of Sonora (HIES). The criteria for inclusion comprised being of 7 to 18 years of age, a T1D diagnose as established according to the American Diabetes Association criteria ${ }^{1}$, a positive anti-GAD and/or anti-IA-2 autoantibodies result, HbA1C levels $<8 \%$, and an antibiotic-free scheme for at least 3 months prior to the sampling. Subjects presenting chronic and inflammatory gastrointestinal diseases were excluded from the study.

In T1D patients, the insulin dose is currently carefully calculated based on the carbohydrate content of the diet and the physical activity. In general, a high-fiber diet with low glycemic index is recommended ${ }^{30}$. All patients' parents were informed about the nature of the study and were asked to provide written informed consent for their child's participation. All patients had nutritional counseling.

Information about birth delivery mode, breastfeeding, complementary feeding patterns in the first year of life, infections and antibiotic treatments prior to T1D diagnosis was collected in an interview with the parent. Clinical records were revised, and the protocol was approved by the Ethics Committee of the Centre for Food Research and Development and the HIES Learning and Research Board.

Sample collection. Peripheral blood $(2 \mathrm{~mL})$ was collected from all patients and stored in EDTA tubes at $4^{\circ} \mathrm{C}$ for haplotype analysis. Stool samples were obtained from every subject and immediately placed on ice for transportation to the laboratory. The DNA extraction was performed within the first $3 \mathrm{~h}$ after collection.

gDNA extraction. Genomic DNA was extracted from whole blood samples using the QIAamp DNA Blood Mini Kit (QIAGEN $\left.{ }^{\circledR}\right)$, following manufacturer's instructions. For stool samples the QIAamp DNA Stool Mini Kit (QIAGEN ${ }^{\circledR}$ ) was used. The concentration and purity were evaluated using a Nanodrop ${ }^{\circledR}$ spectrophotometer (Thermo Scientific, Wilmington, DE, USA). Extracted DNA was stored at $-20^{\circ} \mathrm{C}$.

HLA typing. The HLA DR3-DQ2 and DR4-DQ8 typing was performed from blood gDNA through polymerase chain reaction (PCR) using sequence specific primers ${ }^{31}$ for DRB1*03, DRB1*04, DQA1*0501, DQA1*0301, DQB1*0201 and DQB1*0302/3 alleles (IDT-Integrated DNA Technologies, Tucson-AZ, USA). The amplicons obtained were resolved on $1.8 \%$ agarose gel, stained with GelRed ${ }^{\mathrm{TM}}$ (Biotium Inc., Hayward, CA, USA) and visualized under ultraviolet light (Molecular Imager ${ }^{\circledR}$ BioRad, Hercules, CA, USA). The allele HLA frequencies in both clinical groups were calculated using the direct counting method.

Microbiota determination. The V4 region of the bacterial 16S rRNA gene was amplified from fecal gDNA with 357F/926R primers containing the A and B adaptors from 454 Life Sciences for pyrosequencing, and a unique 12 base pair error correcting Golay barcode, allowing multiplexing of samples in one single run. Replicate amplicons were pooled and purified using the UltraClean -htp 96 well PCR clean up kit (MO BIO). The concentration of samples was measured and samples were combined in equimolar ratios into a single tube. Pyrosequencing was carried out on a 454 Life Sciences Genome Sequencer FLX instrument (Roche) by the Human
Genome Sequencing Center at Baylor College of Medicine, in Houston, Texas, USA, using their standard protocol.

Bioinformatics and community comparisons. The sequence and microbial communities diversity analysis was performed with the QIIME software package (Quantitative Insights Into Microbial Ecology) $^{32}$. Sequences were removed from the analysis if they were $<200 \mathrm{nt}$, had a quality score $<25$, contained ambiguous characters, contained an uncorrectable barcode, or did not contain the primer sequence. Sequences were assigned to samples by examining their individual 12-nt barcode. Sequences were aligned using PyNAST, clustered into OTUs and taxonomy assigned using the Ribosomal Database Project (RDP) as a reference base. The phylogenetic tree was built with FastTree. QIIME and UniFrac were used for analyses of bacterial communities and group comparisons $s^{33}$. Differences between groups were assessed using ANOVA and Tukey-Kramer multiple-comparison test.

1. American Diabetes Association. Standards of medical care in diabetes 2013. Diabetes Care. 36, S11-S66 (2013).

2. Fowler, M. J. Diabetes: magnitude and mechanisms. Clinical Diabetes. 25, 25-28 (2007).

3. Tollefsen, S. et al. Structural and functional studies of trans-encoded HLA-DQ2.3 (DQA1*03:01/DQB1*02:01) protein molecule. J. Biol. Chem. 287, 13611-13619 (2012).

4. Okada, H., Kuhn, C., Feillet, H. \& Bach, J. F. The 'hygiene hypothesis' for autoimmune and allergic diseases: an update. Clin. Exp. Immunol. 160, 1-9 (2010).

5. Patelarou, E. et al. Current evidence on the associations of breastfeeding, infant formula, and cow's milk introduction with type 1 diabetes mellitus: a systematic review. Nutr. Rev. 70, 509-519 (2012).

6. Spagnuolo, I. et al. The case for virus-induced type 1 diabetes. Curr. Opin. Endocrinol. Diabetes Obes. 20, 292-298 (2013).

7. Abela, A. G. \& Fava, S. Association of incidence of type 1 diabetes with mortality from infectious disease and with antibiotic susceptibility at a country level. Acta Diabetol. DOI:1007/s00592-013-0464-z (2013)

8. Muirhead, C. R., Cheetham, T. D., Court, S., Begon, M. \& McNally, R. J. Q. How do childhood diagnoses of type 1 diabetes cluster in time? PLOS ONE 8, e60489 (2013).

9. Brugman, S. et al. Antibiotic treatment partially protects against type 1 diabetes in the Bio-Breeding diabetes-prone rat. Is the gut flora involved in the development of type 1 diabetes? Diabetologia 49, 2105-2108 (2006).

10. Giongo, A. et al. Toward defining the autoimmune microbiome for type 1 diabetes. ISME J. 5, 82-91 (2011).

11. de Goffau, M. C. et al. Fecal microbiota composition differs between children with $\beta$-cell autoimmunity and those without. Diabetes 62, 1238-1244 (2013).

12. Murri, M. et al. Gut microbiota in children with type 1 diabetes differs from that in healthy children: a case-control study. BMC Med. 11, 46 (2013).

13. Vaarala, O. Is the origin of type 1 diabetes in the gut? Immunol. Cell Biol. 90, 271-276 (2012)

14. Oliver-Krasinski, J. M. \& Stoffers, D. A. On the origin of the $\beta$ cell. Genes Dev. 22, 1998-2021 (2008).

15. Kumar, N., Kaur, G., Tandon, N., Kanga, U. \& Mehra, N. K. Genomic evaluation of HLA-DR3 + haplotypes associated with type 1 diabetes. Ann. N. Y. Acad. Sci. 1283, 91-96 (2013)

16. Vadheim, C. M. et al. Different HLA haplotypes in mexican americans with IDDM. Diabetes Care 12, 497-500 (1989).

17. Neu, J. \& Rushing, J. Cesarean versus vaginal delivery: long-term infant outcomes and the hygiene hypothesis. Clin. Perinatol. 38, 321-331(2011).

18. Boerner, B. P. \& Sarvetnick, N. E. Type 1 diabetes: role of intestinal microbiome in humans and mice. Ann. N. Y. Acad. Sci. 1243, 103-118 (2011).

19. Atkinson, M. A. \& Chervonsky, A. Does the gut microbiota have a role in type 1 diabetes? Early evidence from humans and animal models of the disease. Diabetologia 55, 2868-2877 (2012).

20. Yatsunenko, T. et al. Human gut microbiome viewed across age and geography. Nature 486, 222-227 (2012).

21. Lin, A. et al. Distinct distal gut microbiome diversity and composition in healthy children from Bangladesh and the United States. PLOS ONE 8, e53838 (2013).

22. Wu, G. D. et al. Linking long-term dietary patterns with gut microbial enterotypes. Science 334, 105-108(2011).

23. Karvonen, M. et al. Incidence of childhood type 1 diabetes worldwide. Diabetes Mondiale (DiaMond) Project Group. Diabetes Care 23, 1516-1526 (2000).

24. Enríquez-Leal, M. C. et al. Incidence, clinical characteristics and nutritional status in diabetic Mexican children and adolescents. Interciencia 35, 455-460 (2010).

25. Brown, C. T. et al. Gut microbiome metagenomics analysis suggests a functional model for the development of autoimmunity for type 1 diabetes. PLOS ONE 6, e25792 (2011).

26. Tlaskalova-Hogenova, H. et al. The role of gut microbiota (commensal bacteria) and the mucosal barrier in the pathogenesis of inflammatory and autoimmune diseases and cancer: contribution of germ-free and gnotobiotic animal models of human diseases. Cell. Mol. Immunol. 8, 110-120 (2011).

27. Vaarala, O. Human intestinal microbiota and type 1 diabetes. Curr. Diab. Rep. 13, 1-7 (2013). 
28. Berkes, J. Intestinal epithelial responses to enteric pathogens: effects on the tight junction barrier, ion transport, and inflammation. Gut. 52, 439-451 (2003).

29. Zipris, D. The interplay between the gut microbiota and the immune system in the mechanism of type 1 diabetes. Curr. Opin. Endocrinol. Diabetes Obes. 20, 265-270 (2013).

30. American Diabetes Association. Nutrition recommendations and interventions for diabetes-2006: a position statement of the American Diabetes Association. Diabetes Care 29, 2140-2157(2006).

31. Olerup, O., Aldener, A. \& Fogdell, A. HLA-DQB1 and -DQA1 typing by PCR amplification with sequence-specific primers (PCR-SSP) in 2 hours. Tissue Antigens. 41, 119-134 (1993).

32. Caporaso, J. G. et al. QIIME allows analysis of high-throughput community sequencing data. Nature Methods 7, 335-336 (2010).

33. Lozupone, C. \& Knight, R. UniFrac: a new phylogenetic method for comparing microbial communities. Appl. Environ. Microbiol. 71, 8228-8235 (2005).

\section{Acknowledgments}

The work had partial support from the Mexican Council for Science and Technology (CONACYT), grant S0008-2009-01-115212. Authors are grateful to Dr. V. Mata Haro, from CIAD for her advice on the study design, and MD. G. Garcia Galvan, Pediatric Endocrinologist from HIES, for her support during subjects' recruitment. Thanks also to S.M. Rodriguez Rivera from University of Puerto Rico Department of Biology, for her assistance in sequence analysis and to E. Hyde from Baylor College of Medicine Alkek Center for Metagenomics and Microbiome Research, for her comments to the manuscript.

\section{Author contributions}

M.E.M. and A.M.C. designed and conducted the study, collected samples, analyzed haplotypes, extracted DNA, analyzed data and wrote the main manuscript text, J.F.P. and N.J.A. run the microbiota pyrosequence and suggested data analyses methods and M.D.B. supervised the analysis data and wrote the main manuscript text. All authors reviewed the manuscript.

\section{Additional information}

Supplementary information accompanies this paper at http://www.nature.com/ scientificreports

Competing financial interests: The authors declare no competing financial interests.

How to cite this article: Mejía-León, M.E., Petrosino, J.F., Ajami, N.J., Domínguez-Bello, M.G. \& Calderón de la Barca, A.M. Fecal microbiota imbalance in Mexican children with type 1 diabetes. Sci. Rep. 4, 3814; DOI:10.1038/srep03814 (2014).

This work is licensed under a Creative Commons Attribution 3.0 Unported license. To view a copy of this license, visit http://creativecommons.org/licenses/by/3.0 